\title{
Distribución espacial de artrópodos en agroecosistemas de la cuenca del río Limarí (Región de Coquimbo, Chile) ${ }^{1}$
}

\author{
Jaime Pizarro-Araya², Fermín M. Alfaro³, Rodrigo A. Muñoz-Rivera ${ }^{4}$, \\ Juan E. Barriga ${ }^{5}$, Luis Letelier ${ }^{6}$ y Carlos Tirado ${ }^{7}$
}

\begin{abstract}
RESUMEN
El valle del río Limarí tiene un alto valor productivo desde el punto de vista agrícola dentro del Norte Chico. En los últimos años la expansión de los monocultivos ha permitido el aumento de vulnerabilidades biológicas, entre ellas los artrópodos de importancia económica que representan plagas y agentes zoonóticos. Mediante la revisión de bases de datos históricas, colecciones, literatura y colectas entomológicas se analizó la distribución espacial de la riqueza de artrópodos de importancia económica de la cuenca del Limarí. Se contabilizaron 5.418 registros de artrópodos (arácnidos e insectos), los cuales correspondieron en gran mayoría a insectos y en particular a los órdenes Homoptera y Coleoptera. La riqueza y los registros espaciales se agruparon mayoritariamente entre Ovalle y el Estero de Punitaqui, superficies de mayor actividad agrícola. Se espera que estos antecedentes sirvan como información base para entender la estructura de la riqueza de plagas en áreas agrícolas fuertemente modificadas.
\end{abstract}

Palabras clave: Insectos, Norte Chico, plagas agrícolas, registros espaciales, valles transversales.

\begin{abstract}
The Limari River valley has considerable value in terms of agricultural production within the Norte Chico. The expansion of crop monocultures in recents years has increased biological vulnerabilities, such as the number of economically important arthropods that are considered pests and zoonotic agents. Based on the review of historical data, collections, literature and entomological prospections, we examined the spatial distribution of economically important arthropods in the Limari basin. A total of 5,418 arthropods (arachnids and insects) records were registered, most of them insects, particularly homopterans and coleopterans. The richness and spatial records were concentrated between Ovalle and the Punitaqui estuary, which are areas with higher agricultural activity. We hope these data may serve as a basis to understand the richness structure of pest species in highly modified agricultural areas.
\end{abstract}

Key words: Insects, Norte Chico, agricultural pests, spatial records, transverse valleys.

Agradecemos a Adiel R. Cayo (Servicio Agrícola y Ganadero, División de Protección Agrícola y Forestal) por la gestión y acceso a la información proveniente del SAG. A Mario Elgueta (MNNC) y Danilo Cepeda (MEUC) por las facilidades otorgadas en la consulta de las colecciones. Este estudio fue financiado por los proyectos DIDULS PR15121 y PR17121/VACDDI001 de la Universidad de La Serena, La Serena (Chile) (JPA). Artículo recibido el 1 de febrero de 2018, aceptado el 14 de agosto de 2018 y corregido el 13 de septiembre de 2018.

Laboratorio de Entomología Ecológica / Instituto de Investigación Multidisciplinar en Ciencia y Tecnología, Universidad de La Serena (Chile). E-mail: japizarro@userena.cl

3 Laboratorio de Entomología Ecológica / Instituto de Investigación Multidisciplinar en Ciencia y Tecnología, Universidad de La Serena (Chile). E-mail: fmalfaro@userena.cl

4 Laboratorio de Prospección, Monitoreo y Modelación de Recursos Agrícolas y Ambientales (PROMMRA) / Departamento de Agronomía, Universidad de La Serena (Chile). E-mail: ramunoz@userena.cl

Departamento de Ciencias Agrarias / Universidad Católica del Maule (Chile). E-mail: colneotrop@yahoo.com

- Centro de Investigación en Recursos Naturales y Sustentabilidad, Universidad Bernardo O'Higgins / Instituto de Ciencias Biológicas, Universidad de Talca (Chile). E-mail: lealg79@gmail.com

Departamento de Química y Biología / Universidad de Atacama (Chile). E-mail: carlos.tirado@uda.cl 
En el Chile semiárido, la zona del Norte Chico comprende la franja entre los $27^{\circ}$ y $32^{\circ} \mathrm{S}$, abarcando las regiones de Atacama y Coquimbo, y se caracteriza por la presencia de una depresión intermedia interrumpida por cordones montañosos que dan origen a valles transversales que se extienden desde la cordillera de los Andes hasta el Océano Pacifico (DGA, 1986). Tanto los valles de Copiapó $\left(27-28^{\circ} \mathrm{S}\right)$ y Huasco $\left(28-29^{\circ} \mathrm{S}\right)$ en Atacama como los valles de Elqui (30 $\left.\mathrm{S}\right)$, Limarí $\left(31^{\circ}\right.$ S) y Choapa $\left(32^{\circ} \mathrm{S}\right)$ en Coquimbo, corresponden a una matriz semiárida por presentar precipitaciones escasas y dispersas en el tiempo, con ríos de régimen permanente de carácter mixto (DGA, 1986; Ulloa \& Ortiz, 1989; Romero et al., 1998).

Entre los valles transversales del semiárido, se destaca el valle del Río Limarí, considerado el más importante foco económico en términos agroalimentarios (INE, 2007); con actividades productivas secundarias como la agricultura de pequeña escala, la capricultura y la pequeña minería (Cortés, 2016). Actualmente la superficie del valle del Limarí está conformada por cultivos de tipo forrajeros (25.456 ha), frutícolas (20.151 ha), vitícolas (8.353 ha) y hortícolas (4.753 ha) (INE, 2007).

Gran parte de la producción agrícola del valle tiene como destino mercados extranjeros (i.e., EE.UU., Japón, Europa) con exigencias fitosanitarias internacionales (FAO, 2000). Con respecto a los cultivos frutícolas los de mayor importancia económica, debido a las exportaciones internacionales, están representados principalmente por vides de mesa $(7.321,7 \mathrm{ha})$, paltos $(4.128,0$ ha), olivos (2.511,2 ha) y mandarinos $(1.573,4 \mathrm{ha})$, que, junto a otros frutales presentes en el valle, representan el 46,9"\% de los frutales del Norte Chico (INE, 2007; CIREN, 2015). Debido a esto, el control de plagas agrícolas es un factor importante en las exportaciones agrícolas, ya que algunas especies de artrópodos pueden afectar los niveles productivos de los agroecosistemas y a la vez ser causal de rechazo en los mercados de destino (Moore-Tello, 2009).

La dinámica de la biodiversidad en los agroecositemas estaría influenciada por la diversidad de la vegetación, la permanencia de diversos cultivos, la intensidad del manejo y el grado de aislamiento del agroecosistema de la vegetación natural (Altieri \& Nicholls, 2007), y recientemente la sequia (Cortés, 2016, Molinos-Senante et al., 2016). Estos aspectos pueden repercutir a su vez en la dinámica de los ensambles de plagas y enemigos naturales, cuya colonización, establecimiento y crecimiento poblacional estarían en función del grado de modificación del paisaje (Knapp \& Řezáč, 2015).

Debido a esta dinámica fluctuante del paisaje y la alta variabilidad temporal de los cultivos, la distribución espacial de muchas especies de artrópodos sería inestable en el tiempo, factor que condicionaría las acciones de control y manejo tanto de plagas como enemigos naturales en los agroecosistemas (Mazzi \& Dorn, 2012; Zhao et al., 2013; Schellhorn et al., 2014). Una primera aproximación para estudiar estos procesos a escala local es el estudio de los patrones de diversidad y la distribución de la riqueza de estos organismos dentro de los paisajes agrícolas productivos, aspectos no estudiados para la cuenca del río Limarí (Pizarro-Araya et al., 2009; Alfaro et al., 2011). Frente a la escasa información que analiza la riqueza de especies de artrópodos de importancia económica y cómo esta se distribuye dentro de la cuenca del Río Limarí y sus principales subcuencas hidrográficas, en objetivo del presente estudio, fue analizar la distribución espacial de la riqueza de artrópodos de importancia económica de la cuenca del Río Limarí. 


\section{Materiales y métodos}

\section{Área de estudio}

El área de estudio fue definida en base a las tres cuencas asociadas o influenciadas por el Río Limarí; esto es, la propia cuenca del Río Limarí, la Cuenca Costera de los ríos Elqui y Limarí, y finalmente, la cuenca costera de los ríos Limarí y Choapa. Los límites de estas tres cuencas fueron definidos utilizando las capas geográficas del Instituto Geográfico Militar a escala 1:250.000 (Figura $\mathrm{N}^{\circ}$ 1). En esta área predominan suelos de tipo entisol, aridisol e inseptisol, en los que se puede observar algún grado de influencia de la vegetación (Morales, 2006). El clima presente es de tipo estepario, que varía en relación a la altitud desde estepa con nubosidad abundante en la costa a estepa fría de montaña (Novoa \& López, 2001). Las precipitaciones presentan una media anual que supera los $300 \mathrm{~mm}$ en las zonas del sector cordillerano y entre $60-240 \mathrm{~mm}$ en sectores de menor elevación cercanos a la costa (DMC, 2001). La temperatura anual se caracteriza por ser homogénea en las zonas costeras y con oscilaciones térmicas en los valles interiores y en la región cordillerana (DMC, 2001). La vegetación de las zonas interiores no recibe influencia marina, por lo que es de tipo matorral estepario interior (Gajardo, 1993; Squeo et al., 2001).

Figura $N^{\circ}$ 1. Localización geográfica de la cuenca del Río Limarí (Región de Coquimbo, Chile) y registros espaciales de artrópodos de importancia económica utilizados en el estudio.

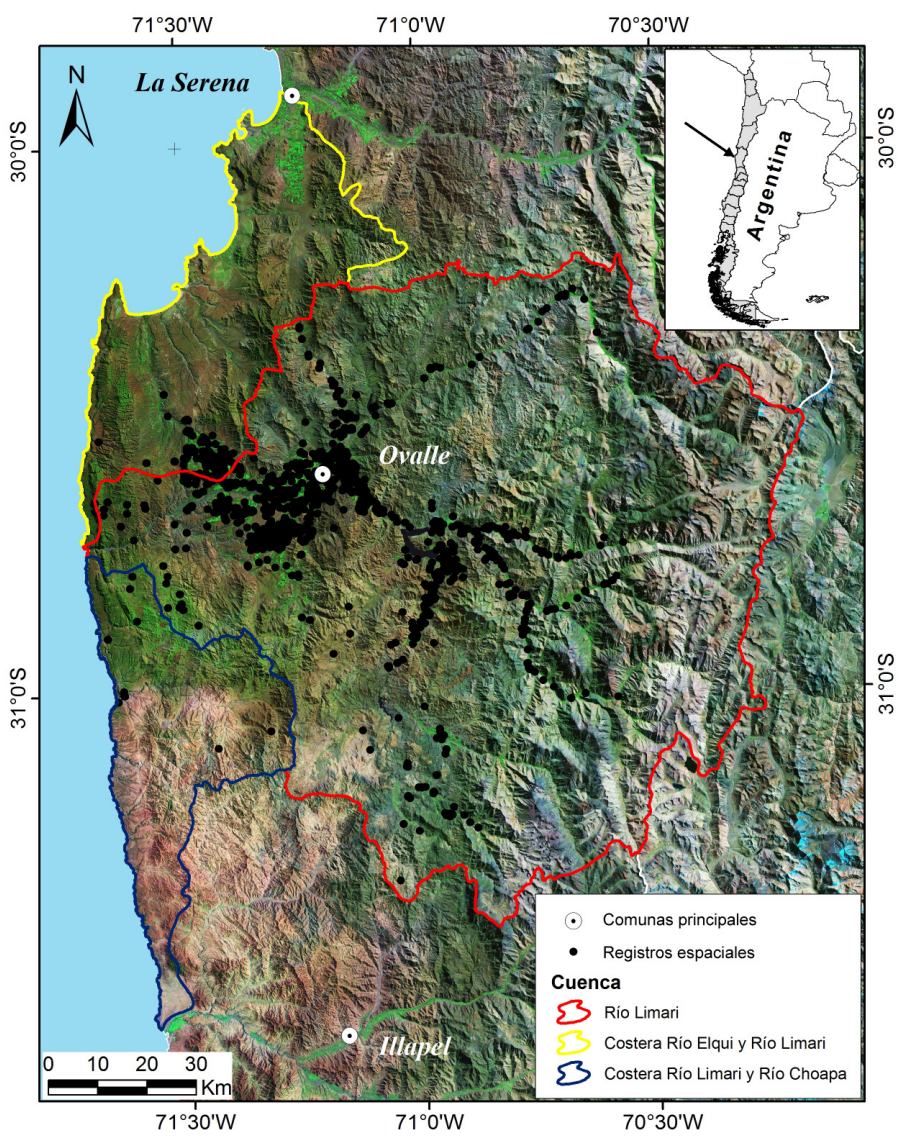




\section{Métodos de captura y colecta de información}

Para analizar la distribución espacial de la riqueza de artrópodos de importancia económica de la cuenca del Limarí se realizó una recopilación de registros distribucionales de artrópodos de importancia económica a partir de la revisión de material de referencia de las siguientes colecciones entomológicas: Juan Enrique Barriga Collection (JEBC); Laboratorio de Entomología Ecológica, Universidad de La Serena, La Serena, Chile (LEULS); Museo Entomológico Luis Peña, Departamento de Sanidad Vegetal, Facultad de Ciencias Agronómicas, Universidad de Chile, Santiago, Chile (MEUC); Museo Nacional de Historia Natural, Santiago, Chile (MNNC). Por otra parte, el Servicio Agrícola y Ganadero de Chile (SAG) proporcionó los registros de capturas entomológicas de la provincia del Limarí efectuados durante los años 2009-2015. Finalmente, estos registros fueron complementados con datos distribucionales señalados en literatura, prospecciones manuales, capturas con red entomológica y uso de luz ultravioleta (UV), realizadas entre los meses de junio y octubre del 2015. El material capturado fue limpiado y conservado en alcohol (70\%) hasta el momento de su procesamiento y montaje. Todo el material recolectado está depositado en la colección del Laboratorio de Entomología Ecológica de la Universidad de La Serena (LEULS). Para la determinación taxonómica de Acari, se siguió a Krantz (1978); para Insecta se siguió a Barriga (1990, 2018), Barriga et al. (1993), Artigas (1994), Ripa \& Rodríguez (2000), Arancibia (2002), Estay \& Bruna (2002), Rojas (2005), Elgueta \& Marvaldi (2006), Vidal \& Guerrero (2007), Castro da Costa (2010), Chiappa (2012), Solervicens (2014), Moore \& Vidal (2015) y González (2017).

\section{Análisis de la información espacial}

Todas las localidades de muestreo fueron referenciadas geográficamente mediante GPS (Etrex-Personal Navigator, Garmin). Para la confección de mapas, la base de datos fue espacializada en el sistema proyectado UTM, huso 19S, datum WGS84. Se utilizó imágenes de satélite LandSat (Chávez, 1996; Chander \& Markham, 2003) y cartografía de apoyo del Instituto Geográfico Militar a escala 1:250.000 (IGM, 1986) para definir el área de estudio y corregir registros respecto de su ubicación espacial. Para realizar los análisis espaciales de la riqueza y el número de registros totales de los artrópodos de importancia económica, se procedió a construir una grilla de $5 \mathrm{~km}$ de radio, procediéndose posteriormente a cruzar la base de datos de registro de especies con la grilla, para así obtener el número total de registros de artrópodos por cada celda o el número de especies (valores únicos) por cada celda.

\section{Distribución espacial de artrópodos en la cuenca del Limarí}

Se contabilizó un total de 5.418 registros de artrópodos (arácnidos e insectos) para la cuenca del Río Limarí, de los cuales el 90,6\% (4.907 registros) fueron para insectos, mientras que sólo 511 registros lo fueron para arácnidos (Figura $N^{\circ} 2$ ). La riqueza total de artrópodos se concentró en áreas bajo el sistema embalse La Paloma y localidades alrededor de la comuna de Ovalle, las que representan importantes terrenos de producción agrícola (Figura $\mathrm{N}^{\circ} 3 \mathrm{a}$ ). Menores niveles de riqueza fueron observados en los principales valles de la cuenca del Río Limarí ubicados sobre el sistema Paloma y que correspondieron principalmente a las comunas de Río Hurtado, Montepatria, Tulahuén y Combarbalá (Figura N 3a). La mayoría de los registros espaciales se distribuyó en un bajo número de cuadrículas, las que correspondieron a terrenos agrícolas de la comuna de 
Ovalle y adyacentes al curso del río Limarí (Figura $\mathrm{N}^{\circ} 3 \mathrm{~b}$ ). Al igual que la distribución espacial de la riqueza de especies, los registros espaciales fueron escasos en las principales subcuencas de las comunas de Río Hurtado, Montepatria, Tulahuén y Combarbalá (Figura $N^{\circ} 3 b$ ). El mayor número de registros para Insecta se observó en los órdenes Homoptera (51,1\%) (principalmente en Aleyrodidae, Pseudococcidae, Aphididae, Diaspididae y Coccidae), Coleoptera $(20,3 \%)$ (principalmente en Coccinellidae, Curculionidae y Chrysomelidae), Thysanoptera (7,1\%) (Thripidae) y Hemiptera $(4,0 \%)$ (Figura $\left.N^{\circ} 2\right)$. Algunas familias como Blattidae (Blattodea), Aderidae, Cantharidae y Cleridae (Coleoptera), Pygidicraniidae (Dermaptera), Cecidomyiidae y Muscidae (Diptera) estuvieron representadas por escasos registros dentro de la cuenca. Por otra parte, Arachnida estuvo representada sólo por Acari y Araneae, siendo las familias Tetranychidae, Tenuipalpidae y Eriophyidae las que presentaron un mayor número de registros espaciales. Familias con un bajo número de registros para la cuenca fueron Anystidae, Ascidae, Eupodidae y Tydeidae.

Figura $\mathrm{N}^{\circ}$ 2. Número de registros espaciales (GPS) para los órdenes de artrópodos de importancia económica de la cuenca del Río Limarí (Región de Coquimbo, Chile). Números entre parentésis representan el porcentaje de acuerdo al total de registros.

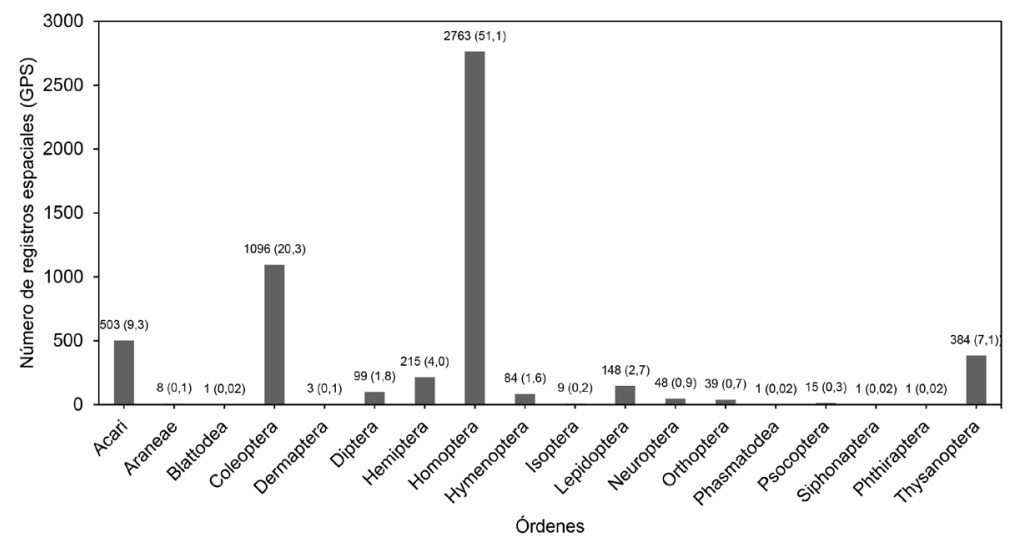

Figura $\mathrm{N}^{\circ} 3$. Mapa de la región de estudio y distribución de las cuadrículas de $5 \mathrm{~km}$ de radio. a) riqueza de artrópodos de importancia económica; b) registros espaciales de artrópodos de importancia económica. Los colores representan intervalos de valores para la riqueza y registros totales dentro de las cuadrículas.

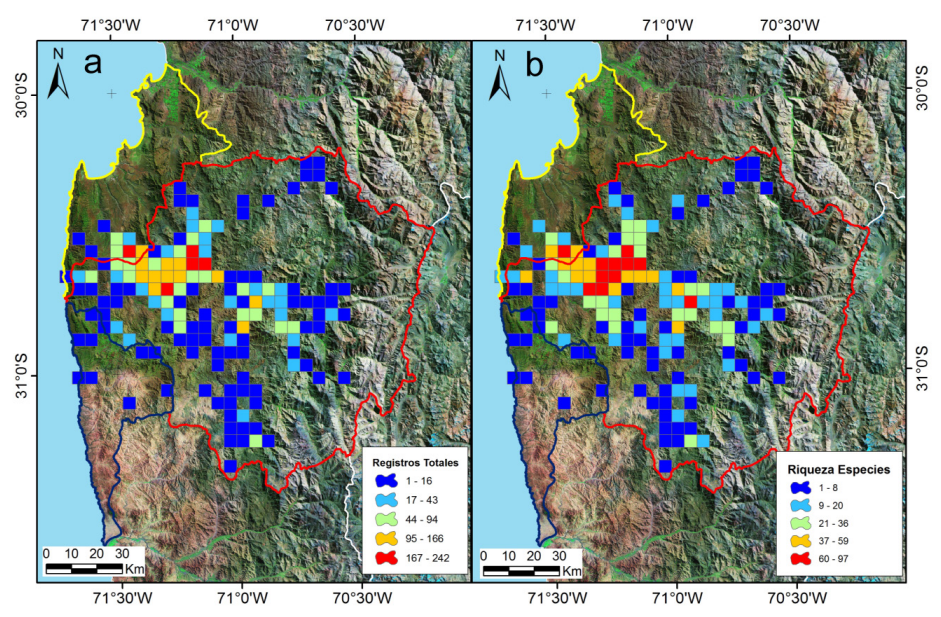


Algunos grupos de amplia distribución dentro de la cuenca fueron los géneros Aleurothrixus Quaintance \& Baker (Homoptera: Aleyrodidae) y Tetranychus Dufour (Acari: Tetranychidae), los cuales presentaron numerosos registros espaciales distribuidos principalmente en las comunas de Ovalle (Río Limarí), Montepatria (ríos Mostazal y Huatulame) y Combarbalá. Algunos géneros como Parlatoria Targioni (Homoptera: Diaspididae) mostraron una distribución restringida a ciertos valles de la cuenca pertenecientes a Montepatria y Tulahuén (Figura $N^{\circ} 4$ ).

Figura $\mathrm{N}^{\circ} 4$. Distribución de los registros totales para los principales géneros (Aleurothrixus, Liriomyza, Parlatoria, Proeulia) de artrópodos de importancia agrícola de la cuenca del Limarí (Región de Coquimbo, Chile). Se indican comunas principales.

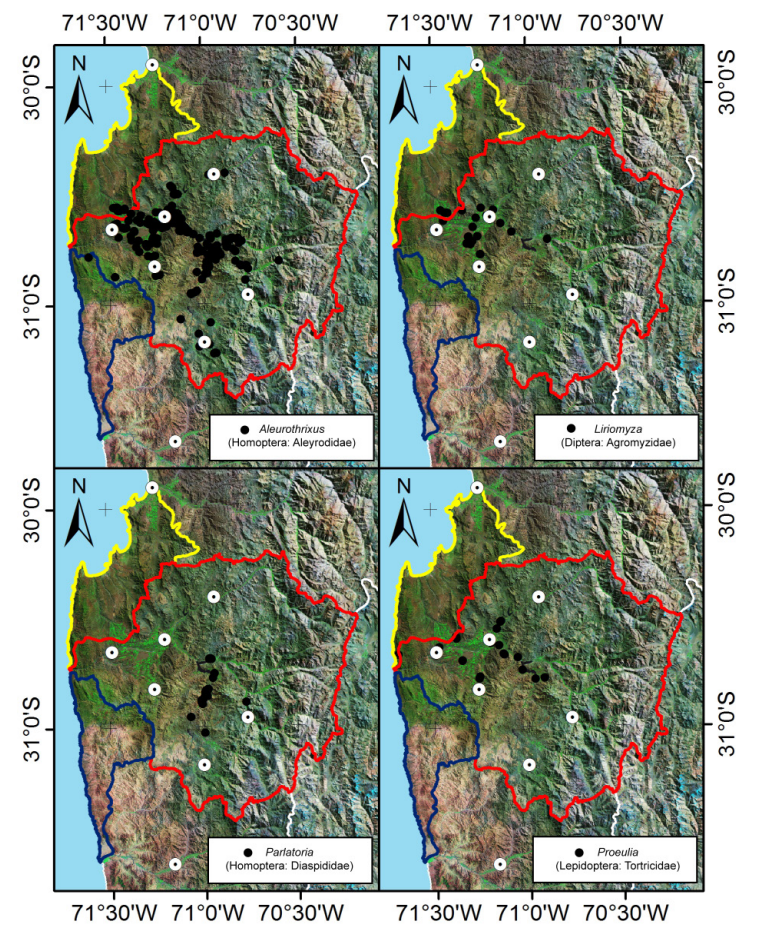

El estudio abarcó una amplia superficie dentro de la cuenca del río Limarí (ver Figura $N^{\circ} 1$ ), en donde se distribuyen diversos cultivos agrícolas (e.g., frutales, hortalizas, cultivos forzados, forestales). Tanto la riqueza como los registros espaciales mostraron una distribución asociada a las principales superficies de explotaciones agrícolas de la cuenca del Limarí. Los mayores niveles de riqueza y registros espaciales se observaron en terrenos agrícolas próximos a la comuna de Ovalle, lo que grafica que las prospecciones realizadas se han concentrado históricamente en aquellas superficies de mayor actividad agrícola como la comuna de Ovalle y aguas abajo 
hacia el Estero de Punitaqui (DGA, 2004; CIREN, 2015). Por otra parte, áreas con baja riqueza y escasos registros espaciales estuvieron asociadas a las subcuencas de los ríos Hurtado, Mostazal y Grande, las cuales poseen una menor superficie agrícola en la cuenca del Limarí (DGA, 2004; CIREN, 2015). Los grupos de mayor distribución de la cuenca fueron Homoptera, Coleoptera, Thysanoptera y Hemiptera, los que comprenden especies plagas asociadas a los principales cultivos del Limarí (e.g., vides, paltos, cítricos) como son los géneros Aleurothrixus Quaintance \& Baker (Homoptera: Aleyrodidae), Pseudococcus Westwood (Homoptera: Pseudococcidae), Naupactus Dejean (Coleoptera: Curculionidae) y Tetranychus Dufour (Acari: Tetranychidae) (ver Figura № 4). Entre las plagas secundarias registradas, se identificaron a los coleópteros Naupactus xanthographus Dejean, 1821 y Platyaspistes glaucus Farhaeus (Curculionidae) (ver Figura N 7a, 7b); los ortópteros Trimerotropis ochraceipennis (Blanchard, 1851) (Acrididae) y Conometopus sulcaticollis (Blanchard, 1851) (Ommexechidae) (ver Figura $N^{\circ} 7 \mathrm{c}, 7 \mathrm{~d}$ ), y las avispas Polistes buyssoni Brèthes, 1909 y Polistes dominulus (Christ, 1791) (Vespidae); grupos de amplia distribución en la cuenca del Limarí (ver Figura $\left.N^{\circ} 7 e, 7 f\right)$.

Figura $N^{\circ} 5$. Distribución de los registros totales para los principales géneros (Lithraeus, Naupactus, Tetranychus, Pseudococcus) de artrópodos de importancia agrícola de la cuenca del Limarí (Región de Coquimbo, Chile). Se indican comunas principales.

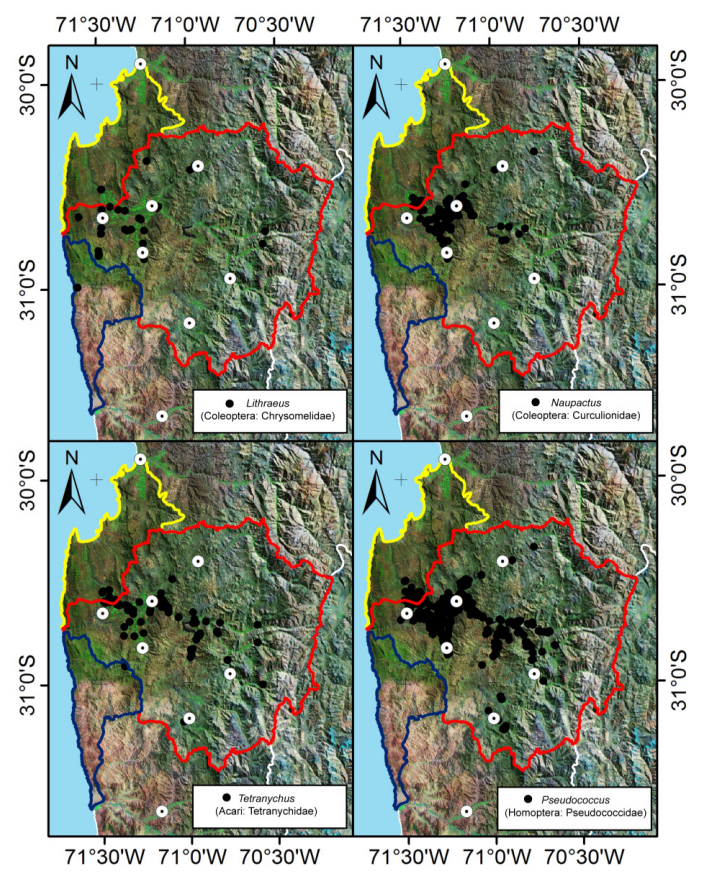


Figura $\mathrm{N}^{\circ}$ 6. Distribución de los registros totales para los principales géneros de artrópodos de importancia forestal (Micrapate, Neotermes) y médico-veterinaria (Mepraia, Triatoma) de la cuenca del Limarí (Región de Coquimbo, Chile). Se indican comunas principales.

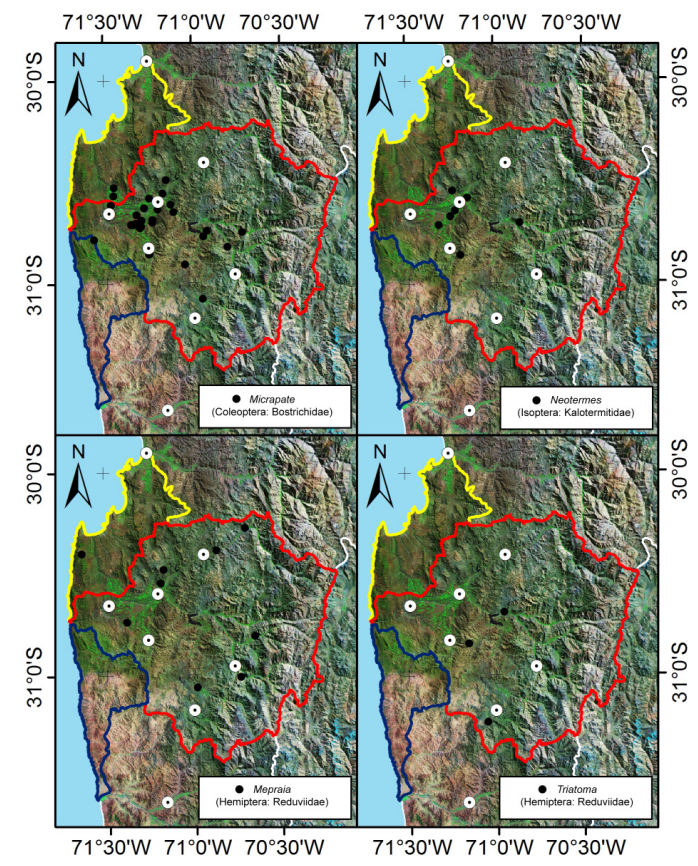

Figura $N^{\circ}$ 7. a) Vista lateral de Naupactus xanthographus Dejean, 1821 (Coleoptera: Curculionidae). b) Vista dorsal de Platyaspistes glaucus Farhaeus, 1840 (Coleoptera: Curculionidae). c) Vista lateral de Trimerotropis ochraceipennis (Blanchard, 1851) (Orthoptera: Acrididae). d) Vista lateral de Conometopus sulcaticollis (Blanchard, 1851) (Orthoptera: Ommexechidae). e) Nido de Polistes buyssoni Brèthes, 1909 (Hymneoptera: Vespidae). f) Nido de Polistes dominulus (Christ, 1791) (Hymneoptera: Vespidae).

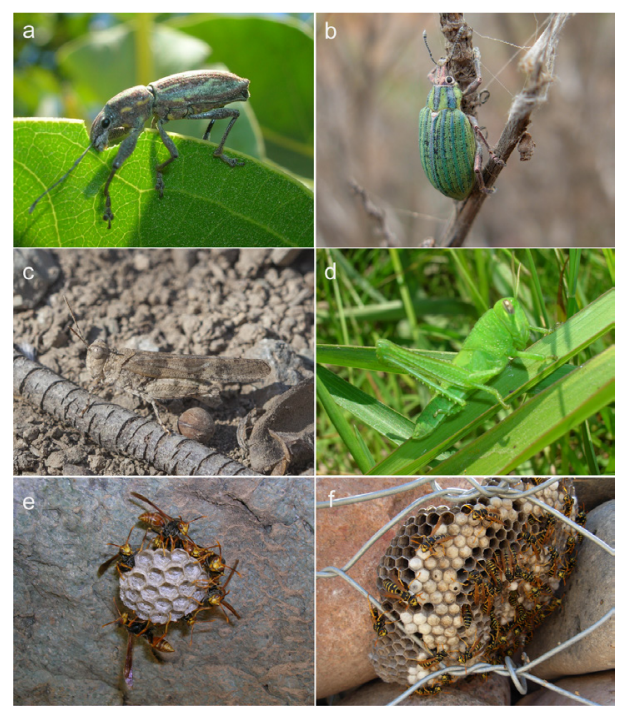


En los últimos años se ha observado una expansión creciente de los cultivos hacia laderas de secano, lo que ha transformado paisajísticamente la matriz vegetal de la cuenca del Limarí. Estos cambios espaciales pueden traer consecuencias en la dinámica poblacional tanto de especies plagas como controladoras; procesos que pueden ser estudiados comprendiendo la distribución y la riqueza de estos grupos de artrópodos de importancia económica. Se espera que el presente estudio permita trabajar en este sentido, sirviendo como información base para desarrollar programas de monitoreo y control de potenciales plagas a nivel local dentro de un sistema agrícola fuertemente modificado en los últimos años.

\section{Conclusiones}

La riqueza total registrada en el área de estudio fue mayor a la documentada para la cuenca del Elqui. Tanto la riqueza como los registros espaciales mostraron una distribución agrupada entre la comuna de Ovalle y aguas abajo hacia el Estero de Punitaqui, superficies de mayor actividad agrícola en la cuenca del Río Limarí; mientras que áreas con menor riqueza y menores registros espaciales correspondieron a las subcuencas de los ríos Hurtado, Mostazal y Grande, con menor superficie de terrenos agrícolas. Los grupos de artrópodos de mayor distribución en la cuenca fueron los géneros Aleurothrixus (Homoptera: Aleyrodidae), Pseudococcus (Homoptera: Pseudococcidae), Naupactus (Coleoptera: Curculionidae) y Tetranychus (Acari: Tetranychidae); que comprenden especies plagas asociadas a los principales cultivos de la cuenca, como vides, paltos y cítricos. Gran parte de los registros de potenciales plagas analizados en este trabajo fueron registrados en superficies agrícolas que históricamente han desarrollado explotaciones agrícolas intensivas.

\section{Referencias bibliográficas}

ALFARO, F.M.; PIZARRO-ARAYA, J.; CEPEDA-PIZARRO, J. y BODINI, A. Composición y distribución del ensamble de Orthoptera (Insecta) en una cuenca árida del norte-centro de Chile. Revista de la Facultad de Ciencias Agrarias, 2011, Vol. 43, N 1, p. 97-110.

ALTIERI, M.A. y NICHOLLS, C.I. Diversidad vegetal y estabilidad de las poblaciones de insectos en los agroecosistemas. En: ALTIERI, M.A. y NICHOLLS, C.I. (editores). Biodiversidad y manejo de plagas en agroecosistemas. Editorial Icaria, Barcelona, España. 2007, p. 41-52.

ARANCIBIA, A.A. Catastro de plagas y enfermedades aéreas presentes en cítricos de la Provincia de Limarí. La Serena: Tesis para optar al título de Ingeniero Agrónomo, Escuela de Agronomía, Facultad de Ciencias, Universidad de La Serena, 2002.

ARTIGAS, J.N. Entomología económica. Insectos de interés agrícola, forestal, médico y veterinario. Concepción: Ediciones Universidad de Concepción, 1994, Vols. I y II.

BARRIGA, J.E. Parásitos y depredadores de larvas de Cerambycidae y Buprestidae (Coleoptera) de Chile. Revista Chilena de Entomología, 1990, Vol. 18, p. 57-59. 
BARRIGA, J.E. Coleoptera Neotropical, 2018. Disponible en Internet:

http://coleoptera-neotropical.org/paginas/2_PAISES/Chile/TENEBRIONOIDEA/teneb_ch.html

BARRIGA, J.E.; CURKOVIC, T.; FICHET, T.; HENRÍQUEZ, J.L. y MACAYA, J. Nuevos antecedentes de coleópteros xilófagos y plantas hospederas en Chile, con una recopilación de citas previas. Revista Chilena de Entomología, 1993, Vol. 20, p. 65-91.

CASTRO DA COSTA, D. Guía de elementos básicos para el monitoreo y la detección de chanchitos blancos (Pseudococcus viburni) para implementar MIP acorde a los requerimientos BPA. Santiago de Chile: Innova Chile Corfo, FDF, 2010.

CHANDER, G. \& MARKHAM, B.L. Revised Landsat 5 TM radiometric calibration procedures and post-calibration dynamic ranges. IEEE Transactions on Geoscience and Remote Sensing, 2003, Vol. 41, № 11, p. 2674-2677.

CHAVEZ, P.S. Image-based atmospheric corrections-revisited and improved. Photogrammetric Engineering \& Remote Sensing, 1996, Vol. 62, № 9, p. 1025-1036.

CHIAPPA, E. Especies de Vespidae y Sphecidae (Hymenoptera) de Valparaíso, Chile: diagnóstico de la distribución regional. Revista Chilena de Entomología, 2012, Vol. 37, p. 5-16.

CENTRO DE INFORMACIÓN DE RECURSOS NATURALES (CIREN). Catastro Frutícola. Principales Resultados. IV Región de Coquimbo/Julio 2015. Santiago de Chile: CIREN, 2015.

CORTÉS, M.E.C. Drought, environmental degradation, work and education: a brief comment on the current reality of agricultural communities in the Limari Province, Chile. Idesia, 2016, Vol. 34, $N^{\circ} 4$, p. 73-76.

DIRECCIÓN GENERAL DE AGUAS (DGA). Diagnóstico y clasificación de los cursos y cuerpos de agua según objetivos de calidad. Cuenca del Río Limarí. Santiago de Chile: Dirección General de Aguas (DGA), 2004. Disponible en Internet:

http://portal.mma.gob.cl/wp-content/uploads/2017/12/Limari.pdf

ELGUETA, M. y MARVALDI, A.E. Lista sistemática de las especies de Curculionoidea (Insecta: Coleoptera) presentes en Chile, con su sinonimia. Boletín del Museo Nacional de Historia Natural, 2006, Vol. 55, p. 113-153.

ESTAY, P. y BRUNA, A. Insectos, ácaros y enfermedades asociadas al tomate en Chile. Santiago de Chile: Instituto de Investigaciones Agropecuarias, , 2002.

ORGANIZACIÓN DE LAS NACIONES UNIDAS PARA LA AGRICULTURA Y LA ALIMENTACIÓN (FAO). Las negociaciones comerciales multilaterales sobre la agricultura III: Acuerdo sobre la Aplicación de Medidas Sanitarias y Fitosanitarias y Acuerdo sobre Obstáculos Técnicos al Comercio. Roma: FAO, 2000.

GAJARDO, R. La vegetación natural de Chile. Clasificación y distribución geográfica. Santiago de Chile: Editorial Universitaria, 1993. 
GONZÁLEZ, F.G. Lista actualizada de Coccinellidae de Chile, 2017. Disponible en Interent: http:// coccinellidae.cl/paginasWebChile/PDFs/Lista\%20Coccinellidae\%20de\%20Chile\%2030\%20 06\%202014.pdf

INSTITUTO GEOGRÁFICO MILITAR DE CHILE (IGM). Carta La Serena. 1:250.000. Santiago de Chile: IGM, 1986.

INSTITUTO NACIONAL DE ESTADÍSTICAS (INE), Censo Agropecuario y Forestal 2007. Santiago de Chile: INE, 2007. Disponible en Internet:

http://www.ine.cl/canales/chile_estadistico/censos_agropecuarios/censo_agropecuario_07.php

KNAPP, M. \& ŘEZÁČ, M. Even the smallest non-crop habitat islands could be beneficial: distribution of carabid beetles and spiders in agricultural landscape. PLoS One, 2015, Vol. 10, № 4, p. e0123052.

KRANTZ, G.W. A manual of Acarology. Oregon: Oregon State University, Corvallis, 1978.

MAZZI, D. \& DORN, S. Movement of insect pests in agricultural landscapes. Annals of Applied Biology, 2012, Vol. 160, p. 97-113.

MOLINOS-SENANTE, M.; DONOSO, G. \& SALA-GARRIDO, R. Are participants in markets for water rights more efficient in the use of water than non-participants? A case study for Limari Valley (Chile). Environmental Science and Pollution Research, 2016, Vol. 23, No 11, p. 10665-10678.

MOORE, T. \& VIDAL, P. Los Bupréstidos de Chile. Ediciones Universidad Católica de Chile, Santiago, Chile, 2015.

MOORE-TELLO, C. Estatus cuarentenario de Pseudococcidae para mercados de fruta de exportación de Chile. Servicio Agrícola y Ganadero (SAG), División Protección Agrícola y Forestal, Santiago de Chile, 2009.

MORALES, L., CANESSA, F., MATTAR, C., ORREGO, R. \& MATUS, F. Caracterización y zonificación edáfica y climática de la Región de Coquimbo, Chile. Revista de la Ciencia del Suelo y Nutrición Vegetal, 2006, Vol. 6, № 3, p. 52-74.

NOVOA, J.E., \& LÓPEZ, D. IV Región: El escenario geográfico físico. En: SQUEO, F.A., ARANCIO, G. y GUTIÉRREZ, J.R. (editores). Libro Rojo de la Flora Nativa y de los Sitios Prioritarios para su Conservación: Región de Coquimbo. Ediciones Universidad de La Serena, La Serena, Chile. 2001, p. $13-28$.

PIZARRO-ARAYA, J., CEPEDA-PIZARRO, J., BARRIGA, J.E. \& BODINI, A. Biological vulnerability in the Elqui valley (Coquimbo región, Chile) to economically important arthropods. Ciencia e Investigación Agraria, 2009, Vol. 36, № 2, p. 215-228.

RIPA, R., \& RODRÍGUEZ, F. Plagas de cítricos, sus enemigos naturales y manejo. Instituto de Investigaciones Agropecuarias, Santiago, Chile, 2000. 
ROJAS, S. Control biológico de plagas en Chile. Historia y avances. Colección Libros Instituto de Investigaciones Agropecuarias, Santiago, Chile, 2005.

SCHELLHORN, N.A., BIANCHI, F.J.J.A. \& HSU, C.L. Movement of entomophagous arthropods in agricultural landscapes: links to pest suppression. Annual Review of Entomology, 2014, Vol. 59, p. 559-581.

SOLERVICENS, J. Coleópteros de la Reserva Nacional Río Clarillo, en Chile Central: taxonomía, biología y biogeografía. Corporación Nacional Forestal (CONAF), Santiago, Chile, 2014.

SQUEO, F.A., ARANCIO, G. \& GUTIÉRREZ, J.R. Libro Rojo de la Flora Nativa y de los Sitios Prioritarios para su Conservación: Región de Coquimbo. Ediciones Universidad de La Serena, La Serena, Chile, 2001.

VIDAL, P., \& GUERRERO, M. Los Tenebrionidae de Chile. Ediciones Universidad Católica de Chile, Santiago, Chile, 2007.

ZHAO, Z.H., HUI, C., OUYANG, F., LIU, J.H., GUAN, X.Q., HE, D.H. \& GE, F. Effects of inter-annual landscape change on interactions between cereal aphids and their natural enemies. Basic and Applied Ecology, 2013, Vol. 14, p. 472-479. 